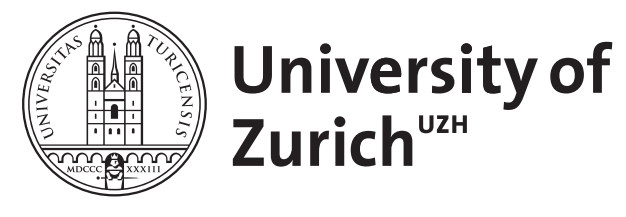

Zurich Open Repository and Archive

University of Zurich

University Library

Strickhofstrasse 39

CH-8057 Zurich

www.zora.uzh.ch

Year: 2016

\title{
Über Arbeit von Ärzten und anderen
}

Wolff, Eberhard

Posted at the Zurich Open Repository and Archive, University of Zurich ZORA URL: https://doi.org/10.5167/uzh-131733

Journal Article

Published Version

Originally published at:

Wolff, Eberhard (2016). Über Arbeit von Ärzten und anderen. Schweizerische Ärztezeitung (SÄZ), 97(8):310. 


\section{Über Arbeit von Ärzten und anderen}

\section{Eberhard Wolff}

PD Dr. rer. soc., Redaktor Kultur, Geschichte, Gesellschaft

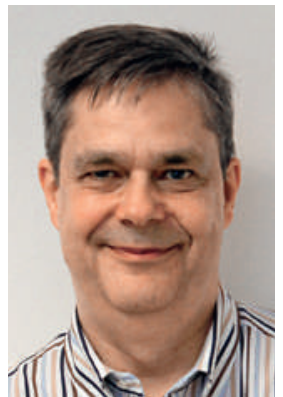

Wir können in diesem Blatt immer wieder lesen, dass sich die (ambulante) ärztliche Arbeitswelt zurzeit ändert: mehr Teilzeittätigkeit, weniger Arbeitspensum, weniger Einzelpraxen [1, 2]. Ist das Teil eines generellen Trends in der Arbeitswelt oder läuft es dem entgegen? Ein neuer Sammelband aus dem Schweizerischen Sozialarchiv mit dem doppeldeutigen Titel Ausser Betrieb [3] hilft, Antworten auf diese Frage zu finden.

In ihrer anregenden Einleitung betonen die Herausgebenden, Brigitta Bernet und Jakob Tanner, dass die stabile und kollektivvertraglich regulierte «Normalerwerbsarbeit» von qualifizierten Industrie-Angestellten aus der Wirtschaftswunder-Zeit immer mehr von flexibleren Arbeitsverhältnissen abgelöst wird. Statt Vollzeit-Arbeit im gleichen Betrieb gibt es jetzt zunehmend Fristverträge, Teilzeit oder (Schein-)Selbstän-

\section{Auch Ärzte fühlen sich heute weniger als} früher deckungsgleich mit ihren Berufen, ihrer Arbeit, ihrer Praxis.

digkeit, in ständiger Verfügbarkeit oder an wechselnden Arbeitsstellen. Unterbrochen von Auszeiten oder Arbeitslosigkeit. Kurz: Wir arbeiten flexibler, dafür aber unsicherer, wir arbeiten quantitativ vielleicht weniger, dafür aber entgrenzter. Und das als Mischung aus Wollen und Müssen.

Im Arztberuf ist schon der Ausgangspunkt ein anderer. Niedergelassene machen als Kleinunternehmer kaum die Erfahrungen geregelter «Normalerwerbsarbeit». Und die angestellten Spitalärztinnen und -ärzte haben die kollektivvertraglich regulierte Arbeitswelt der Wirtschaftswunderzeit verzögert und erst teilweise betreten. Siehe etwa ihre Arbeitszeiten.

Dennoch folgt ein Teil der Ärzteschaft einigen der allgemeinen Trends. Einen Tag die Woche freiwillig bei den Kindern oder im eigenen Garten sein zu wollen, das gibt es bei den Ärzten - und mehr noch Ärztinnen - genauso wie bei Softwareingenieuren. Wir haben uns in den letzten Jahren allgemein ein Stück weit aus dem früheren Modell einer betrieblich orientierten "Arbeitsgesellschaft» gelöst, so Bernet und Tanner. Der arbeitswütige Börsenbroker ist heute mehr Kuriosum als Rollenmodell. Auch Ärzte fühlen sich heute weniger als früher deckungsgleich mit ihren Berufen, ihrer Arbeit, ihrer Praxis. In einer Gruppenpraxis oder einem Ärztenetzwerk zu arbeiten bietet mehr Möglichkeiten, neben dem
Beruf auch noch Ehepartner oder Töfffahrerin und nicht «Herr/Frau Doktor» zu sein.

Gleichzeitig gibt es Unterschiede zum allgemeinen Trend. In einer flexibilisierten Arbeitswelt müssen Menschen heute, so Bernet und Tanner, immer mehr wie «Unternehmer ihrer Selbst» denken. Niedergelassene Mediziner waren die längste Zeit so etwas wie «Ich-AGs». Immer mehr wollen jetzt weniger wie (Einzel-)Unternehmer denken müssen. Sie lassen sich im «Betrieb» einer Arztpraxis anstellen. Oder sie gründen Gemeinschaftspraxen bzw. Ärztenetzwerke. Dann sind sie zumindest eine «Wir-AG».

Heute fliessen Arbeit und Nicht-Arbeit immer mehr ineinander. Werbetexter oder Versicherungsagenten nehmen gewollt oder gedrängt ihre Arbeit per Laptop und Smartphone mit nach Hause. Das bringt Freiheiten, aber auch die Allgegenwart der Arbeit - natürlich auch unter Medizinern. Immer mehr von ihnen wollen sich zurzeit jedoch aus der ständigen Verfügbarkeit etwa der klassischen Landarztrolle lösen und suchen mehr «Normalerwerbsarbeit».

Laufen Ärzte mit oder gegen den Trend zunehmend flexibilisierter Arbeit? Ich habe den Eindruck, als ob die hier beschriebenen Mediziner jeweils die «Wollen»Bereiche der flexibilisierten wie auch der traditionellen Arbeitswelt im Blick haben. Sie suchen geregelte, überschaubare Arbeitszeiten, die gleichzeitig flexibel genug sind, den individuellen Zeitwünschen zu entsprechen. Die unangenehmeren «Müssen»-Teile der heute flexibilisierteren Arbeit, vor allem die berufliche Unsicherheit und Abhängigkeit, können sie aufgrund des Ärztemangels derzeit wohl leichter umgehen.

Ich vermute, dass sich hier eine Variante von Arztsein entwickelt (eine weiblichere?), deren Berufsverständnis sich ebenso vom traditionellen, zeitlich fremdbestimmten Landarzt wie auch vom zeitlich genauso fremdbestimmten (angehenden) Chefarzt unterscheidet. Nicht "Ausser Betrieb», sondern ausserhalb klassischer Arztrollen. Ich kenne einige, die auf dem Weg dorthin sind, und solche, die davon träumen.

\section{Literatur}

1 Maurer L. Löst die Gemeinschaftspraxis die klassische Einzelpraxis ab? Schweiz Ärztezeitung. 2014;95(25):971-2.

2 Hostettler S, Kraft E. Frauen- und Ausländeranteil nehmen kontinuierlich zu. Schweiz Ärztezeitung. 2015;96(13):462-9.

3 Bernet B, Tanner J (eds.). Ausser Betrieb. Metamorphosen der Arbeit in der Schweiz. Zürich: Limmat-Verlag; 2015. 